\title{
Fuzzy MIMO detector for MC-CDMA systems with carrier frequency offset over multipath fading channels
}

\author{
Juinn-Horng Deng ${ }^{*}$ and Shu-Min Liao
}

\begin{abstract}
A multistage fuzzy minimum output energy (MOE) detector is proposed for multiple input multiple output (MIMO) multi-carrier code-division-multiple access (MC-CDMA) uplink systems with carrier frequency offset (CFO) over multipath fading channels. The first stage of the receiver uses a novel MIMO receiver model with offset symbols to achieve a fuzzy CFO-constrained MOE detector that suppresses multiple access interference (MAl) and minimizes cancellation of the desired signal. To suppress noise and to enhance signal reception after the fuzzy MOE detector, a signal subspace projection and minimum mean square error weight combiner is proposed to enhance signal-to-interference-plus-noise ratio and bit error rate performances. Simulation results show that the proposed MIMO detector outperforms conventional MOE detectors and achieves the ideal receiver performance against MAI and CFO effects.
\end{abstract}

Keywords: Multi-carrier CDMA (MC-CDMA), Multiple input multiple output (MIMO), Multiple access interference (MAl), Carrier frequency offset (CFO), Minimum output energy (MOE)

\section{Introduction}

Multi-carrier code-division-multiple access (MCCDMA) systems [1-4] are currently under consideration for application in WiMAX and WiFi systems in future broadband wireless communication systems. The system has recently attracted attention because it combines high spectral efficiency with a robust channel equalizer against multipath fading channels. Like a directsequence CDMA (DS-CDMA) system, MC-CDMA inherently overcomes the intersymbol interference caused by multipath propagation. However, its performance is limited by multiple access interference (MAI) and carrier frequency offset (CFO) [5]. The CFO can cause loss of subcarrier orthogonality, which results in intercarrier interference (ICI). To minimize the CFO effect, the receiver in most uplink MC-CDMA systems [6] must first accurately estimate and compensate for CFO to maintain the orthogonality between multi-carriers. Additionally, the rotated constellation of the desired signal degrades the performance of MC-CDMA systems $[7,8]$.

\footnotetext{
* Correspondence: jh.deng@saturn.yzu.edu.tw

Communication Research Center and Department of Communication Engineering, Yuan Ze University, Chungli, Taoyuan 32003, Taiwan
}

Popular frequency offset estimators are widely reported in the literature [9-13]. For example, the maximum likelihood estimator is the optimal estimator under additive white Gaussian noise. Existing frequency offset estimators typically assume little or no interference. Unfortunately, frequency offset cannot be estimated accurately when MAI is strong. Therefore, an MC-CDMA receiver must efficiently suppress MAI before performing frequency offset compensation. More importantly, MAI suppression must be independent of frequency offset.

The efficient minimum output energy (MOE) multiuser detector developed earlier for use in suppressing MAI in MC-CDMA systems [14] is designed to minimize the entire output energy of the receiver by constraining the spreading code sequence. However, the ICI effect of the CFO in MC-CDMA systems produces a mismatched spreading code signature, which substantially deteriorates MOE detector performance due to the signal cancellation effect [15]. One proposed solution is the use of subspace MOE detectors [16,17], which are designed to project the weight vector of the MOE scheme onto the signal subspace of the received observation data. However, the subspace MOE still involves 
the problem of the desired signal cancellation to degrade the system performance. Besides, a well-known technique for improving the link quality for wireless communication in the multiple input multiple output (MIMO) scheme is space-time block coding (STBC) [18]. For example, the WiMAX system [19] adopts the spatial multiplexing and diversity schemes of the matrices $\mathrm{A}$ and B MIMO schemes [20] used for user uplink communication. Therefore, this study proposes the use of MIMO STBC MC-CDMA system with multiuser uplink communication for the CFO and multipath fading channel environments. Additionally, since the conventional space-time block decoding scheme in [18] cannot be used for the MAI, ICI, and multipath interference scenarios, the proposed MIMO STBC receiver with fuzzy constrained MOE detector suppresses interference and effectively detects desired signals.

Moreover, to acquire spatial diversity, this study develops a robust MIMO fuzzy MOE detector to suppress MAI, to prevent signal cancellation caused by the CFO effect, and to acquire the multipath and spatial diversity gains. The receiver design procedure is the following. First, a novel MIMO received data model with offset symbol is proposed to prevent the CFO from causing symbol detection failure in the STBC MC-CDMA system. Second, the offset MIMO receiver uses a simple spectral peak search scheme for roughly estimating CFO. Although the CFO estimate need not be accurate, it should lead to the correct CFO region. Next, a MIMO fuzzy MOE detector is then proposed for identifying the novel eigen-based signature with MIMO fuzzy CFO constraint used to suppress MAI. Finally, to maximize suppression of noise and reception of signals after the MIMO fuzzy MOE detector, use of the signal subspace projection and minimum mean square error (MMSE) techniques are proposed to enhance signal-to-interference-plus-noise ratio (SINR) and bit error rate (BER) performances. Simulation results confirm that the proposed MIMO detector has sufficient robustness to suppress CFO and MAI effects and that it outperforms conventional detectors. The rest of this article is organized as follows: Section 2 presents the data model and the ideal receiver. Section 3 then presents the robust MIMO multistage fuzzy MOE detector. Section 4 analyzes the computational complexity of the proposed MIMO multistage fuzzy MOE detector. Next, Section 5 summarizes the simulation results. Finally, Section 6 concludes the study.

\section{Notation}

The following notation is used. Bold uppercase letters, e. g., A, are used to denote matrices, bold lowercase letters, e.g., a, are used to denote column vectors, and non-bold letters in italics, e.g., $a$ or $A$, are used to denote scalar values. The symbols $\mathbf{A}^{H}, \mathbf{A}^{T}, \mathbf{A}^{\prime \prime}$, and $\operatorname{tr}(\mathbf{A})$ denote the transpose-conjugate, the transpose, the complex conjugate, and the trace operations of $\mathbf{A}$, respectively. The $\operatorname{diag}(\mathbf{a})$ represents a diagonal matrix whose diagonal entries are the elements of the vector $\mathbf{a}$.

\section{Data model for MIMO MC-CDMA systems}

Consider an uplink multiuser MIMO MC-CDMA system over multipath channels with frequency offset. Figure 1 is a block diagram of the proposed MIMO MCCDMA transceiver. The MIMO structure in the diagram is the simplest case of two transmitting antennas and two receiving antennas, which can be extended to multiple receiver antennas with increased spatial diversity gain. That is, for the scenario of subscriber user with two transmit antennas and base station with $N_{R}$ receiver antennas, the proposed scheme can simultaneously be processed by each receiver. Next, the combiner can then collect the equalized signal from different receivers, which enhances detection performance. In the transmitter, assume a $K$ active user transmission in the uplink MIMO MC-CDMA system, each user is assigned a unique spreading code in the frequency domain such that the complex transmitted signal vectors with $N \times 1$ dimension $\left(\mathbf{s}_{k}^{(m)}(i), \mathbf{s}_{k}^{(m)}(i+1)\right), \quad m=1,2$ of the $k$ th user for the $i$ th and $(i+1)$ th data symbol after the STBC can be expressed by

$$
\begin{aligned}
& \mathbf{s}_{k}^{(1)}(i)=\sigma_{k}^{(1)} d_{k}(i), \mathbf{Q}^{H} \mathbf{c}_{k} \\
& \mathbf{s}_{k}^{(1)}(i+1)=-\sigma_{k}^{(1)} d_{k}^{*}(i+1) \mathbf{Q}^{H} \mathbf{c}_{k} \\
& \mathbf{s}_{k}^{(2)}(i)=\sigma_{k}^{(2)} d_{k}(i+1) \mathbf{Q}^{H} \mathbf{c}_{k}, \\
& \mathbf{s}_{k}^{(2)}(i+1)=\sigma_{k}^{(2)} d_{k}^{*}(i) \mathbf{Q}^{H} \mathbf{c}_{k}
\end{aligned}
$$

where the super-scripts (1) and (2) denote the first and second transmitter antennas, respectively, and $\sigma_{k}^{(1)}=$ $\sigma_{k}^{(2)}=1 / \sqrt{2} \sigma_{k}$ is the transmitted amplitude of two transmitter antennas with total power of $\sigma_{k}^{2}$. The $\mathbf{c}_{k}$ is an $N \times 1$ vector with the spreading code of length $N$, where $\mathbf{c}_{1}$ is the spreading code of the desired user 1 . The $\mathbf{Q}^{H}$ denotes the $N \times N$ orthogonal inverse fast Fourier transform matrix. The $d_{k}(i)$ and $d_{k}(i+1)$ are the $i$ th and $(i+1)$ th data symbols, respectively. Next, to prevent interblock interference, a cyclic prefix (CP) longer than the multipath channel response is then inserted into each transmitted data block. For fixed wireless communication, the channel impulse response (CIR) $L_{k} \times 1$ vector of user $k$ from the $n_{T}$ th transmit antenna to the $n_{R}$ th receive antenna can be modeled as $h_{k}^{\left(n_{R}, n_{T}\right)}=\left[h_{k}^{\left(n_{R}, n_{T}\right)}(0) h_{k}^{\left(n_{R}, n_{T}\right)}(1) \cdots\right.$ $\left.h_{k}^{\left(n_{R}, n_{T}\right)}\left(L_{k}-1\right)\right]^{T}, n_{\mathcal{T}} n_{R}=1$ or 2 , where $L_{k}$ is the channel length. The assumptions are that CIR $h_{k}^{\left(n_{R}, n_{T}\right)}(i), i=$ $0, \ldots, L_{k}-1$ is an independent, identically distributed (i.i. d.) complex Gaussian random variable with zero-mean and 


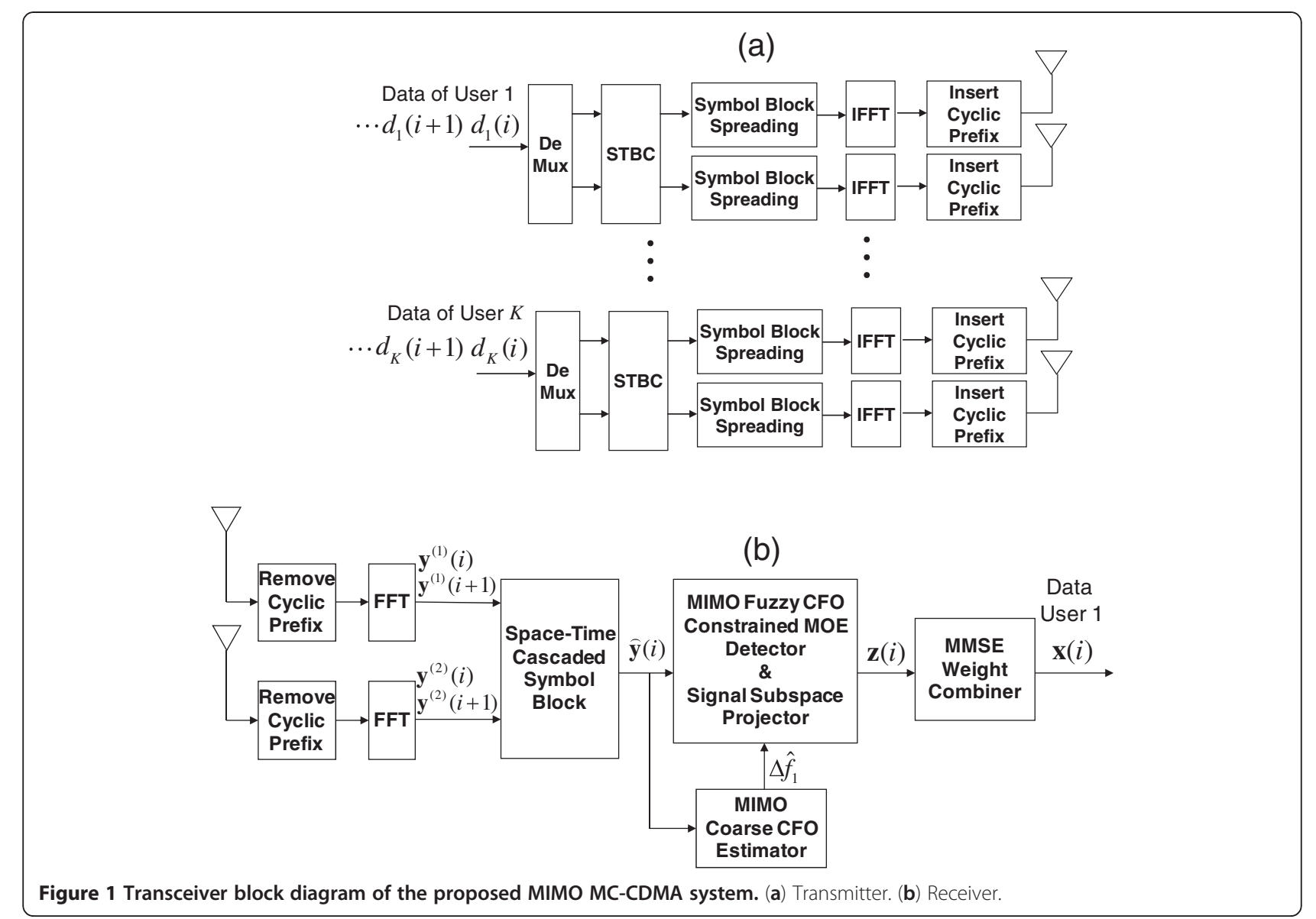

unit-variance and that $\mathbf{h}_{k}^{\left(n_{R}, n_{T}\right)}$ is a quasi-static channel that remains constant during each packet and is independent between packets. Next, let $\Delta \varepsilon_{k}=\Delta f_{k} T_{k}$ denote the unknown normalized CFO of user $k$ between the transmitter and receiver, let $\Delta f_{k}$ denote the CFO of the $k$ th user, and let $T_{k}$ denote the MC-CDMA symbol duration of the $k$ th user [21].

Figure $1 \mathrm{~b}$ is a MIMO receiver block diagram showing that, after discarding $\mathrm{CP}$ and applying fast Fourier transform (FFT), the $i$ th and $(i+1)$ th post-FFT received signal blocks with $N \times 1$ dimension from the first and second receiver antennas in the presence of CFO can be expressed by

$$
\begin{aligned}
& \mathbf{y}^{\left(n_{R}\right)}(i)=\sum_{k=1}^{K} \frac{1}{\sqrt{2}} \sigma_{k} e^{j \phi_{k}(i-1)} \mathbf{G}_{k}\left(\Delta \varepsilon_{k}\right) \\
& \quad \times\left\{d_{k}(i) \mathbf{H}_{k}^{\left(n_{R}, 1\right)} \mathbf{c}_{k}+d_{k}(i+1) \mathbf{H}_{k}^{\left(n_{R}, 2\right)} \mathbf{c}_{k}\right\}+\mathbf{v}^{\left(n_{R}\right)}(i) \\
& \mathbf{y}^{\left(n_{R}\right)}(i+1)=\sum_{k=1}^{K} \frac{1}{\sqrt{2}} \sigma_{k} e^{j \phi_{k} i} \mathbf{G}_{k}\left(\Delta \varepsilon_{k}\right) \\
& \quad \times\left\{-d_{k}^{*}(i+1) \mathbf{H}_{k}^{\left(n_{R}, 1\right)} \mathbf{c}_{k}+d_{k}^{*}(i) \mathbf{H}_{k}^{\left(n_{R}, 2\right)} \mathbf{c}_{k}\right\} \\
& +\mathbf{v}^{\left(n_{R}\right)}(i+1), n_{R}=1,2
\end{aligned}
$$

where $\mathbf{H}_{k}^{\left(n_{R}, n_{T}\right)}=\operatorname{diag}\left\{\mathbf{Q} \mathbf{h}_{k}^{\left(n_{R}, n_{T}\right)}\right\}, n_{T}, n_{R}=1$ or 2 , denotes the $N \times N$ frequency domain channel matrix from the $n_{T}$ th transmit antennas to the $n_{R}$ th receive antenna. The $N \times N$ CFO effect matrix in (2) is modeled by $e^{j \phi_{k}(q)} \mathbf{G}_{k}\left(\Delta \varepsilon_{k}\right), q=i$ - $1, \quad i$, with $\mathbf{G}_{k}\left(\Delta \varepsilon_{k}\right)=\mathbf{Q} \operatorname{diag}\left\{e^{j 2 \pi \Delta \varepsilon_{k} N_{g} / N}, \ldots\right.$, $\left.e^{j 2 \pi \Delta \varepsilon_{k}\left(N_{g}+N-1\right) / N}\right\} \mathbf{Q}^{H}$ and $\phi_{k}=j 2 \pi \Delta \varepsilon_{k}\left(N_{g}+N\right) / N$. The $\mathbf{v}^{\left(n_{R}\right)}(i)$ and $\mathbf{v}^{\left(n_{R}\right)}(i+1), n_{R}=1,2$, denote the $N \times 1$ complex white Gaussian noise with zero mean and covariance matrix $\sigma_{n}^{2} \mathbf{I}_{N \times N}$. Without loss of generality, the signal model in (2) then assumes that user 1 is the desired user and that the others are MAI. Thus, the received signals of the $i$ th and $(i+1)$ th symbol blocks in (2) can be rewritten as

$$
\begin{aligned}
& \mathbf{y}^{\left(n_{R}\right)}(i)=e^{j \phi_{1}(i-1)}\left\{d_{1}(i) \overline{\mathbf{C}}_{1} \overline{\mathbf{h}}_{1}^{\left(n_{R}, 1\right)}+d_{1}(i+1) \overline{\mathbf{C}}_{1} \overline{\mathbf{h}}_{1}^{\left(n_{R}, 2\right)}\right\} \\
& \quad+\mathbf{i}^{\left(n_{R}\right)}(i)+\mathbf{v}^{\left(n_{R}\right)}(i) \\
& \mathbf{y}^{\left(n_{R}\right)}(i+1)=e^{j \phi_{1} i}\left\{d_{1}^{*}(i) \overline{\mathbf{C}}_{1} \overline{\mathbf{h}}_{1}^{\left(n_{R}, 2\right)}-d_{1}^{*}(i+1) \overline{\mathbf{C}}_{1} \overline{\mathbf{h}}_{1}^{\left(n_{R}, 1\right)}\right\} \\
& \quad+\mathbf{i}^{\left(n_{R}\right)}(i+1)+\mathbf{v}^{\left(n_{R}\right)}(i+1),
\end{aligned}
$$

respectively, for $n_{R}=1,2$, where $\overline{\mathbf{C}}_{1}$ is the $N \times L_{1}$ frequency shift spreading code matrix of user 1 due to CFO and multipath delay time, i.e., $\overline{\mathbf{C}}_{1}=\mathbf{G}_{1}\left(\Delta \varepsilon_{1}\right) \mathbf{C}_{1}$ with the $N \times L_{1}$ composite spreading code matrix $\mathbf{C}_{1}=\left[\mathbf{c}_{1,0} \cdots \mathbf{c}_{1, l} \cdots \mathbf{c}_{1, L_{1}-1}\right]$ 
and $\mathbf{c}_{1, l}=\operatorname{diag}\left(\mathbf{c}_{1}\right) \mathbf{q}_{l}$ with the $\mathbf{q}_{l}$ being the $l$ th $N \times 1$ column vector of $\mathbf{Q}$ matrix. Although the original spreading code $\mathbf{c}_{1,0}$ is used in the frequency domain in the MCCDMA system, note that the original spreading code in the CFO and multipath environment is distorted by the CFO and different time delay of multipath effect in the frequency domain. Next, the channel response gain vectors with $N \times$ 1 dimensions are $\overline{\mathbf{h}}_{1}^{\left(n_{R}, n_{T}\right)}=1 / \sqrt{2} \sigma_{1} \mathbf{h}_{1}^{\left(n_{R}, n_{T}\right)}, n_{T}, n_{R}=1$ or 2 . The $N \times 1$ MAI vectors are denoted by $\mathbf{i}^{\left(n_{R}\right)}(i)$ and $\mathbf{i}^{\left(n_{R}\right)}(i+1), n_{R}=1,2$. The conventional STBC system can then be used to construct the orthogonal signal structure and to acquire the diversity gain of the transmitter antenna over the single user in the perfect synchronization scenario. However, in (3) with MAI and CFO effects, the conventional STBC receiver design [18] cannot be used for MAI and CFO scenarios when constructed directly by $\mathbf{y}^{n_{R}}(i)$ and $\mathbf{y}^{n_{R}}(i+1), n_{R}=1,2$. Therefore, the conventional receiver design detects the signal in (3) by first compensating for the frequency offset effect and then using linear weight combiner to suppress the MAI interference. That is, after an ideal frequency offset compensation, the post-FFT received signal vector in (3) can be rewritten as

$$
\begin{aligned}
& \overline{\mathbf{y}}^{\left(n_{R}\right)}(i)=d_{1}(i) \mathbf{C}_{1} \overline{\mathbf{h}}_{1}^{\left(n_{R}, 1\right)}+d_{1}(i+1) \mathbf{C}_{1} \overline{\mathbf{h}}_{1}^{\left(n_{R}, 2\right)} \\
& \quad+\overline{\mathbf{i}}^{\left(n_{R}\right)}(i)+\overline{\mathbf{v}}^{\left(n_{R}\right)}(i) \\
& \overline{\mathbf{y}}^{\left(n_{R}\right)}(i+1)=d_{1}^{*}(i) \mathbf{C}_{1} \overline{\mathbf{h}}_{1}^{\left(n_{R}, 2\right)}-d_{1}^{*}(i+1) \mathbf{C}_{1} \overline{\mathbf{h}}_{1}^{\left(n_{R}, 1\right)} \\
& \quad+\overline{\mathbf{i}}^{\left(n_{R}\right)}(i+1)+\overline{\mathbf{v}}^{\left(n_{R}\right)}(i+1)
\end{aligned}
$$

for $n_{R}=1,2$. The post-FFT received signal in (4) for the $i$ th and $(i+1)$ th symbols can also be cascaded as $\tilde{\mathbf{y}}(i)$ with $4 N \times 1$ vector size:

$$
\begin{aligned}
\tilde{\mathbf{y}}(i) & =\left[\overline{\mathbf{y}}^{(1)^{T}}(i) \overline{\mathbf{y}}^{(1)^{H}}(i+1) \overline{\mathbf{y}}^{(2)^{T}}(i) \overline{\mathbf{y}}^{(2)^{H}}(i+1)\right]^{T} \\
& =d_{1}(i) \tilde{\mathbf{h}}_{1}+d_{1}(i+1) \tilde{\mathbf{h}}_{2}+\tilde{\mathbf{i}}(i)+\tilde{\mathbf{v}}(i)
\end{aligned}
$$

where $\tilde{\mathbf{h}}_{1}$ and $\tilde{\mathbf{h}}_{2}$ are the $4 N \times 1$ composite channel vectors, i.e.,

$$
\begin{aligned}
\tilde{\mathbf{h}}_{1}= & \operatorname{diag}\left\{\overline{\mathbf{C}}_{1} \overline{\mathbf{C}}_{1}^{*} \overline{\mathbf{C}}_{1} \overline{\mathbf{C}}_{1}^{*}\right\}\left[\overline{\mathbf{h}}_{1}^{(1,1)^{T}} \overline{\mathbf{h}}_{1}^{(1,2)^{H}} \overline{\mathbf{h}}_{1}^{(2,1)^{T}} \overline{\mathbf{h}}_{1}^{(2,2)^{H}}\right]^{T}, \\
\tilde{\mathbf{h}}_{2}= & \operatorname{diag}\left\{\overline{\mathbf{C}}_{1} \overline{\mathbf{C}}_{1}^{*} \overline{\mathbf{C}}_{1} \overline{\mathbf{C}}_{1}^{*}\right\} \\
& \times\left[\overline{\mathbf{h}}_{1}^{(1,2)^{T}}-\overline{\mathbf{h}}_{1}^{(1,1)^{H}} \overline{\mathbf{h}}_{1}^{(2,2)^{T}}-\overline{\mathbf{h}}_{1}^{(2,1)^{H}}\right]^{T},
\end{aligned}
$$

$\tilde{\mathbf{i}}(i)$ and $\tilde{\mathbf{v}}(i)$ are the $4 N \times 1$ composite MAI and noise vectors, respectively. To recover $d_{1}(i)$ and $d_{1}(i+1)$ from $\tilde{\mathbf{y}}(i)$, a linear receiver can be used to calculate

$$
x_{1}(i)=\mathbf{w}_{1}^{H} \tilde{\mathbf{y}}(i) \text { and } x_{1}(i+1)=\mathbf{w}_{2}^{H} \tilde{\mathbf{y}}(i)
$$

where $\mathbf{w}_{1}$ and $\mathbf{w}_{2}$ are the $4 N \times 1$ weight vectors. The ideal maximum SINR (MSINR) weight vector can be chosen by [22], i.e.,

$$
\mathbf{w}_{1}=\mathbf{R}_{i n, 1}^{-1} \tilde{\mathbf{h}}_{1} \text { and } \mathbf{w}_{2}=\mathbf{R}_{i n, 2}^{-1} \tilde{\mathbf{h}}_{2}
$$

where the $4 N \times 4 N$ covariance matrices are

$$
\begin{aligned}
\mathbf{R}_{i n, 1}= & E\left\{( d _ { 1 } ( i + 1 ) \tilde { \mathbf { h } } _ { 2 } + \tilde { \mathbf { i } } ( i ) + \tilde { \mathbf { v } } ( i ) ) \left(d_{1}(i+1) \tilde{\mathbf{h}}_{2}\right.\right. \\
& \left.+\tilde{\mathbf{i}}(i)+\tilde{\mathbf{v}}(i))^{H}\right\}
\end{aligned}
$$

and

$$
\begin{aligned}
\mathbf{R}_{i n, 2}= & E\left\{( d _ { 1 } ( i ) \tilde { \mathbf { h } } _ { 1 } + \tilde { \mathbf { i } } ( i ) + \tilde { \mathbf { v } } ( i ) ) \left(d_{1}(i) \tilde{\mathbf{h}}_{1}\right.\right. \\
& \left.+\tilde{\mathbf{i}}(i)+\tilde{\mathbf{v}}(i))^{H}\right\} .
\end{aligned}
$$

Thus, the desired signals $d_{1}(i)$ and $d_{1}(i+1)$ can be detected by $x_{1}(i)$ and $x_{1}(i+1)$, respectively.

It is noted that the ideal MSINR receiver in (6) is implemented by artificially knowing the desired signal components $d_{1}(i)$ and $d_{1}(i=1)$ in the data, and using the true composite channel vector $\tilde{\mathbf{h}}_{1}$ and $\tilde{\mathbf{h}}_{2}$ to obtain (7). That is, the ideal MSINR receiver is designed to perform the optimum bound of the proposed MIMO MC-CDMA system, which cannot be realized since the desired signal and the true composite channel are the ideal known assumptions. Therefore, in this article, we will propose a robust fuzzy MIMO MC-CDMA receiver with MAI suppression over CFO and multipath fading channel environment.

\section{Robust MIMO MC-CDMA receiver}

Figure $1 \mathrm{~b}$ shows a block diagram of the multistage fuzzy MIMO MC-CDMA receiver. In the first stage of the receiver, a novel received data structure is proposed to overcome the failure of the STBC MC-CDMA system due to CFO. Next, a robust fuzzy CFO-constrained MIMO MOE method with coarse CFO estimation and signal subspace projection is designed to suppress MAI. Finally, the post-MOE output data are constructively combined by MMSE weight vector to extract the symbols transmitted by the desired user.

\section{Novel MIMO receiver model with offset symbols}

Again, consider the scenario of the uplink MIMO MCCDMA receiver data model in (3) in a multipath fading channel with frequency offset. The complex signals $d_{1}(i)=d_{1 I}(i)+j d_{1 Q}(i) \quad$ and $\quad d_{1}(i+1)=d_{1 I}(i+1)+$ 
$j d_{1 Q}(i+1)$ are first substituted into (3) to provide an alternative receiver data model:

$$
\begin{aligned}
\mathbf{y}^{\left(n_{R}\right)}(i)= & e^{j \phi_{1}(i-1)} \overline{\mathbf{C}}_{1}\left[\overline{\mathbf{h}}_{1}^{\left(n_{R}, 1\right)} \overline{\mathbf{h}}_{1}^{\left(n_{R}, 2\right)}\right] \\
& \times\left[\begin{array}{l}
d_{1 I}(i)+j d_{1 Q}(i) \\
d_{1 I}(i+1)+j d_{1 Q}(i+1)
\end{array}\right] \\
& +\mathbf{i}^{\left(n_{R}\right)}(i)+\mathbf{v}^{\left(n_{R}\right)}(i) \\
= & e^{j \phi_{1}(i-1)} \overline{\mathbf{C}}_{1}\left[\overline{\mathbf{h}}_{1}^{\left(n_{R}, 1\right)} \overline{\mathbf{h}}_{1}^{\left(n_{R}, 2\right)}\right] \\
& \times\left\{\mathbf{b}_{1}(i)+\overline{\mathbf{b}}_{1}(i)\right\}+\mathbf{i}^{\left(n_{R}\right)}(i)+\mathbf{v}^{\left(n_{R}\right)}(i)
\end{aligned}
$$

and

$$
\begin{aligned}
\mathbf{y}^{\left(n_{R}\right)}(i+1)= & e^{j \phi_{1} i} \overline{\mathbf{C}}_{1}\left[\overline{\mathbf{h}}_{1}^{\left(n_{R}, 2\right)} \overline{\mathbf{h}}_{1}^{\left(n_{R}, 1\right)}\right] \\
\times & \times\left[\begin{array}{l}
d_{1 I}(i)-j d_{1 Q}(i) \\
-d_{1 I}(i+1)+j d_{1 Q}(i+1)
\end{array}\right] \\
+ & \mathbf{i}^{\left(n_{R}\right)}(i+1)+\mathbf{v}^{\left(n_{R}\right)}(i+1) \\
= & e^{j \phi_{1} i} \overline{\mathbf{C}}_{1}\left[\overline{\mathbf{h}}_{1}^{\left(n_{R}, 2\right)} \overline{\mathbf{h}}_{1}^{\left(n_{R}, 1\right)}\right]\left\{\mathbf{b}_{1}(i)-\overline{\mathbf{b}}_{1}(i)\right\} \\
& +\mathbf{i}^{\left(n_{R}\right)}(i+1)+\mathbf{v}^{\left(n_{R}\right)}(i+1)
\end{aligned}
$$

for $n_{R}=1,2$, where $\mathbf{b}_{1}(i)=\left[d_{1 I}(i) j d_{1 Q}(i+1)\right]^{T}$ and $\overline{\mathbf{b}}_{1}(i)=\left[j d_{1 Q}(i) d_{1 I}(i+1)\right]^{T}$ are the $2 \times 1$ reconstructed transmission signal vectors. The reconstructed $4 N \times 1$ data vector $\widehat{\mathbf{y}}(i)$ over the $i$ th and $(i+1)$ th symbols can then be cascaded by

$$
\begin{aligned}
\widehat{\mathbf{y}}(i)= & {\left[\mathbf{y}^{(1)^{T}}(i) \mathbf{y}^{(1)^{T}}(i+1) \mathbf{y}^{(2)^{T}}(i) \mathbf{y}^{(2)^{T}}(i+1)\right]^{T} } \\
= & e^{j \phi_{1}(i-1)} \tilde{\mathbf{C}}_{1}(\Delta \varepsilon)\left\{\mathbf{H}_{1} \mathbf{b}_{1}(i)+\mathbf{H}_{2} \overline{\mathbf{b}}_{1}(i)\right\} \\
& +\widetilde{\mathbf{i}}(i)+\widehat{\mathbf{v}}(i)
\end{aligned}
$$

where $\tilde{\mathbf{C}}_{1}(\Delta \varepsilon)=\operatorname{diag}\left\{\overline{\mathbf{C}}_{1}, e^{j \phi_{1}} \overline{\mathbf{C}}_{1}, \overline{\mathbf{C}}_{1}, e^{j \phi_{1}} \overline{\mathbf{C}}_{1}\right\}$ is the effective MIMO CFO shift spreading code matrix with $4 N \times$ $4 L_{1}$ dimension and the $4 L_{1} \times 2$ MIMO channels are $\mathbf{H}_{1}=\left[\begin{array}{lll}\overline{\mathbf{h}}_{1}^{(1,1)^{T}} \overline{\mathbf{h}}_{1}^{(1,2)^{T}} \overline{\mathbf{h}}_{1}^{(2,1)^{T}} \overline{\mathbf{h}}_{1}^{(2,2)^{T}} \\ \overline{\mathbf{h}}_{1}^{(1,2)^{T}} \overline{\mathbf{h}}_{1}^{(1,1)^{T}} \overline{\mathbf{h}}_{1}^{(2,2)^{T}} \overline{\mathbf{h}}_{1}^{(2,1)^{T}}\end{array}\right]^{T}$ and

$\mathbf{H}_{2}=\left[\begin{array}{l}\overline{\mathbf{h}}_{1}^{(1,1)^{T}}-\overline{\mathbf{h}}_{1}^{(1,2)^{T}} \overline{\mathbf{h}}_{1}^{(2,1)^{T}}-\overline{\mathbf{h}}_{1}^{(2,2)^{T}} \\ \overline{\mathbf{h}}_{1}^{(1,2)^{T}}-\overline{\mathbf{h}}_{1}^{(1,1)^{T}} \overline{\mathbf{h}}_{1}^{(2,2)^{T}}-\overline{\mathbf{h}}_{1}^{(2,1)^{T}}\end{array}\right]^{T}$. The $\widehat{\mathbf{i}}(i)$ and $\mathbf{n}(i)$ are the MAI and the noise vectors with $4 N \times 1$ dimension, respectively. In (10), the $4 N \times 4 N$ data correlation matrix $\mathbf{R}_{\hat{y} y}$ can be derived by

$$
\begin{aligned}
\mathbf{R}_{\widehat{y y}}= & E\left\{\widehat{\mathbf{y}}(i) \widehat{\mathbf{y}}^{H}(i)\right\} \\
= & \tilde{\mathbf{C}}_{1}(\Delta \varepsilon) \mathbf{H}_{1} E\left\{\mathbf{b}_{1}(i) \mathbf{b}_{1}^{H}(i)\right\} \mathbf{H}_{1}^{H} \tilde{\mathbf{C}}_{1}^{H}(\Delta \varepsilon) \\
& +\tilde{\mathbf{C}}_{1}(\Delta \varepsilon) \mathbf{H}_{2} E\left\{\overline{\mathbf{b}}_{1}(i) \overline{\mathbf{b}}_{1}^{H}(i)\right\} \mathbf{H}_{2}^{H} \tilde{\mathbf{C}}_{1}^{H}(\Delta \varepsilon) \\
& +\mathbf{R}_{\text {in }}=\mathbf{R}_{s}+\mathbf{R}_{i n}
\end{aligned}
$$

where $\mathbf{R}_{i n}=E\left\{(\widetilde{\mathbf{i}}(i)+\widehat{\mathbf{v}}(i))(\widehat{\mathbf{i}}(i)+\widehat{\mathbf{v}}(i))^{H}\right\}$ is the $4 N \times$ $4 N$ MAI-plus-noise correlation matrix. Notably, the two reconstructed symbols $\left(\mathbf{b}_{1}(i), \overline{\mathbf{b}}_{1}(i)\right)$ in (11) involve the orthogonal cross-correlation and the same covariance property, i.e.,

$$
\begin{aligned}
& E\left\{\mathbf{b}_{1}(i) \overline{\mathbf{b}}_{1}^{H}(i)\right\}=\mathbf{0} \\
& E\left\{\mathbf{b}_{1}(i) \mathbf{b}_{1}^{H}(i)\right\}=\left[\begin{array}{cc}
\sigma_{1, I}^{2} & 0 \\
0 & \sigma_{1, Q}^{2}
\end{array}\right]=\frac{\sigma_{1}^{2}}{2} \mathbf{I} \\
& E\left\{\overline{\mathbf{b}}_{1}(i) \overline{\mathbf{b}}_{1}^{H}(i)\right\}=\left[\begin{array}{cc}
\sigma_{1, I}^{2} & 0 \\
0 & \sigma_{1, Q}^{2}
\end{array}\right]=\frac{\sigma_{1}^{2}}{2} \mathbf{I}
\end{aligned}
$$

After substituting (12) into (11), the desired signal correlation matrix $\mathbf{R}_{s}$ with $4 N \times 4 N$ dimension can be rewritten as

$$
\mathbf{R}_{s}=\tilde{\mathbf{C}}_{1}(\Delta \varepsilon)\left\{\frac{1}{2} \sigma_{1}^{2}\left(\mathbf{H}_{1} \mathbf{H}_{1}^{H}+\mathbf{H}_{2} \mathbf{H}_{2}^{H}\right)\right\} \tilde{\mathbf{C}}_{1}^{H}(\Delta \varepsilon)
$$

Since $\mathbf{H}_{1}$ and $\mathbf{H}_{2}$ are CFO-independent, $\mathbf{R}_{s}$ can only be affected by the frequency shift matrix $\tilde{\mathbf{C}}_{1}(\Delta \varepsilon)$. Therefore, this feature can be used for coarse estimation of CFO as described below.

\section{Coarse CFO estimator}

Section 2 showed why the optimum receiver must accurately estimate $\mathrm{CFO}$ and compensate for the $\mathrm{CFO}$ effect to prevent MAI. Here, however, the CFO estimate need not be accurate as required in the proposed fuzzy MOE receiver, but it should indicate the correct $\mathrm{CFO}$ region. A fuzzy frequency offset-constrained MIMO MOE receiver is then proposed for effective suppression of strong MAI. Restated, the frequency offset in the conventional system in (4) requires precise estimation and compensation by the receiver so that the optimum receiver can suppress the MAI. The proposed receiver, however, uses the coarse CFO searcher with signal subspace constraint in (15) to estimate the correct CFO region with residual $\mathrm{CFO}$ in the same manner as a global searcher for CFO parameters. After the coarse CFO estimation, the fine residual CFO parameter can be considered for use in the MOE detector of Section 3.3 to span the effective signal subspace, to acquire the desired signal reception, and to suppress MAI interference. The results of performance tests are discussed in Section 5 . The proposed coarse CFO estimation procedure is performed in the following steps. First, since spreading code $\mathbf{C}_{1}$ is given, the CFO shift spreading code matrix $\tilde{\mathbf{C}}_{1}(\Delta \varepsilon)$ with $4 N \times 4 L_{1}$ dimension can be constructed by the different frequency offset, i.e.,

$$
\begin{aligned}
\tilde{\mathbf{C}}_{1}(\Delta \varepsilon)= & \operatorname{diag}\left\{\mathbf{G}_{1}(\Delta \varepsilon) \mathbf{C}_{1}, e^{j \phi_{1}} \mathbf{G}_{1}(\Delta \varepsilon) \mathbf{C}_{1},\right. \\
& \left.\mathbf{G}_{1}(\Delta \varepsilon) \mathbf{C}_{1}, e^{j \phi_{1}} \mathbf{G}_{1}(\Delta \varepsilon) \mathbf{C}_{1}\right\}
\end{aligned}
$$

where $\mathbf{G}_{1}(\Delta \varepsilon)$ is an $N \times N$ matrix, $\mathbf{C}_{1}$ is an $N \times L_{1}$ matrix, and $\Delta \varepsilon$ is uniformly increased to search for the 
coarse CFO. Next, based on the reconstructed data model in (13) and the CFO shift spreading code matrix in (14), a simple CFO estimator is determined as the solution to the following spectral search problem:

$$
\Delta \hat{\varepsilon}_{1}=\max _{\Delta \varepsilon_{1}} S\left(\Delta \varepsilon_{1}\right) \equiv \operatorname{tr}\left\{\mathbf{U}_{s}^{H} \tilde{\mathbf{C}}_{1}(\Delta \varepsilon) \tilde{\mathbf{C}}_{1}^{H}(\Delta \varepsilon) \mathbf{U}_{s}\right\}
$$

where the $4 N \times 2 K$ matrix $\mathbf{U}_{s}$ contains $2 K$ dominant eigenvectors of $\mathbf{R}_{\hat{y} \hat{y}}$ in (11), which span the signal subspace. Note that the signal subspace $\mathbf{U}_{\mathrm{s}}$, which is obtained from the signal covariance matrix $\mathbf{R}_{\mathrm{s}}$ in (11) and (13), can be used to provide the desired signal subspace and to limit the interference subspace to converge the desired coarse CFO estimate for the spectral search in (15). That is, in (15), for the estimated frequency offset $\Delta \varepsilon_{1}$ approaching the CFO of desired user, $\tilde{\mathbf{C}}_{1}(\Delta \varepsilon)$ produces a spectral lobe at $\Delta \varepsilon_{1}$ and suppresses MAI outside $\Delta \varepsilon_{1}$. Therefore, if the spectral peak error in the spectrum satisfies the condition $\left|S_{i}\left(\Delta \varepsilon_{1}\right)-S_{i-1}\left(\Delta \varepsilon_{1}\right)\right| /$ $S_{i}\left(\Delta \varepsilon_{1}\right)<0.1$, the $\Delta \varepsilon_{1}$ is varied with the $i$ th searching iteration by using $\Delta \varepsilon_{1}$ as the coarsely estimated CFO of the desired user.

\section{Fuzzy CFO-constrained MIMO MOE detector}

Based on the previous CFO estimator, the CFO can roughly be estimated by the simple spectral searcher in (15). Next, after the reconstructed data is subjected to the CFO effect $\tilde{\mathbf{C}}_{1}(\Delta \varepsilon)$ as described in (10), a fuzzy CFO-constrained algorithm with coarse CFO initialization is proposed to suppress MAI and to collect multipath energy for the linear MOE detector. The optimization problem for the MIMO MOE can be expressed by

$$
\begin{aligned}
& \min _{\mathbf{w}} E\left\{\left|\mathbf{w}^{H} \widehat{\mathbf{y}}(i)\right|^{2}\right\} \equiv \mathbf{w}^{H} \mathbf{R}_{\hat{y} y} \mathbf{w} \\
& \text { subject to }: \mathbf{w}^{H} \mathbf{E}_{1}=\mathbf{i}_{i}^{T} \quad i=1,2, \cdots, D
\end{aligned}
$$

where $\mathbf{i}_{i}$ is the $i$ th column of the identity matrix $\mathbf{I}$ and $D$ is the constraint size. Matrix $\mathbf{E}_{1}$, which is the MIMO fuzzy CFO-constrained signature matrix with coarse CFO $\Delta \hat{\varepsilon}_{1}$ initiation, is then chosen from the dominant eigenvectors of the distorted signature matrix with a dense set of CFO $\hat{\varepsilon}_{n}=\Delta \hat{\varepsilon}_{1}+\varepsilon_{n}, n=1,2, \ldots, N_{t}$, i.e.,

$$
\begin{aligned}
\mathbf{R}_{\varepsilon} & =\frac{1}{N_{t}} \sum_{n=1}^{N_{t}} \tilde{\mathbf{C}}_{1}\left(\hat{\varepsilon}_{n}\right) \tilde{\mathbf{C}}_{1}^{H}\left(\hat{\varepsilon}_{n}\right) \\
& =\sum_{i=1}^{4 N} \lambda_{i} \mathbf{e}_{i} \mathbf{e}_{i}^{H} \\
\mathbf{E}_{1} & =\left[\mathbf{e}_{1}, \mathbf{e}_{2} \cdots, \mathbf{e}_{D}\right]
\end{aligned}
$$

where $\left\{\boldsymbol{\lambda}_{\boldsymbol{i}}\right\}$ and $\left\{\mathbf{e}_{\boldsymbol{i}}\right\}$ are eigenvalues and $4 N \times 1$ eigenvectors of the $4 N \times 4 N \boldsymbol{R}_{\varepsilon}$ matrix. The shift matrix $\tilde{\mathbf{C}}_{1}\left(\hat{\varepsilon}_{n}\right)=$
$\operatorname{diag}\left\{\mathbf{G}_{1}\left(\hat{\varepsilon}_{n}\right) \mathbf{C}_{1}, e^{j \hat{\phi}_{1}} \mathbf{G}_{1}\left(\hat{\varepsilon}_{n}\right) \mathbf{C}_{1}, \mathbf{G}_{1}\left(\hat{\varepsilon}_{n}\right) \mathbf{C}_{1}, e^{j \hat{\phi}_{1}} \mathbf{G}_{1}\left(\hat{\varepsilon}_{n}\right) \mathbf{C}_{1}\right\}$ involves the phase shift $\hat{\phi}_{1}=j 2 \pi \hat{\varepsilon}_{n}\left(N_{g}+N\right) / N$. Since the $D$ is the dominant mode with large eigenvalues in (17) and $N_{t}$ is the number of frequencies in the dense set of frequencies $\hat{\varepsilon}_{n}$, the $N_{t}$ shift matrices $\tilde{\mathbf{C}}_{1}\left(\hat{\varepsilon}_{n}\right)$ span an effective rank of $D$ for the fuzzy CFO-constrained matrix $\mathbf{E}_{1}$ with $4 N \times D$ dimension. The $4 N \times 1$ weight vector $\mathbf{w}_{i}$ of the MIMO fuzzy CFO-constrained MOE detector in (16) can be determined by

$$
\begin{aligned}
& \mathbf{w}_{i}=\mathbf{R}_{\hat{y} y}^{-1} \mathbf{E}_{1}\left(\mathbf{E}_{1}^{H} \mathbf{R}_{\hat{y} y}^{-1} \mathbf{E}_{1}\right)^{-1} \mathbf{i}_{i} \\
& \mathbf{W}=\left[\mathbf{w}_{1} \mathbf{w}_{2} \cdots \mathbf{w}_{i} \cdots \mathbf{w}_{D}\right]=\mathbf{R}_{y y}^{-1} \mathbf{E}_{1}\left(\mathbf{E}_{1}^{H} \mathbf{R}_{y y}^{-1} \mathbf{E}_{1}\right)^{-1}
\end{aligned}
$$

where $\mathbf{W}$ is the $4 N \times D$ weight matrix of fuzzy MOE detector. Notably, the MIMO fuzzy CFO constraint enables the proposed MIMO MOE detector to avoid the problem of sensitivity to signature mismatch arising in the conventional MOE [7], which the signature mismatch effect can significantly degrade system performance. Moreover, in (17), the dominant eigenvectors can span the desired signal subspace containing the mismatch signatures induced by CFO effect. Therefore, the new constrained signature $\mathbf{E}_{1}$ in (16) can consist of the dominant eigenvectors to collect the desired signal energy and to suppress other user interference.

\section{Signal subspace projection of MOE weight}

Fuzzy MOE processing gives the proposed receiver sufficient robustness to suppress strong MAI. However, the fuzzy CFO constraint increases noise in the post-MOE received data. Therefore, the constrained MOE weight matrix in (18) is projected onto the signal subspace, which can minimize the effects of noise and improve system performance. The $4 N \times D$ projected weight matrix $\mathbf{W}_{\mathrm{s}}$ can be expressed by

$$
\mathbf{W}_{s}=\mathbf{U}_{s} \mathbf{U}_{s}^{H} \mathbf{W}
$$

where $\mathbf{U}_{s}$ is the signal subspace of $\mathbf{R}_{\hat{y} \hat{y}}$ as in (15).

\section{MMSE weight combiner}

After the above multistage processing procedures eliminate MAI, only the desired signal is retained in the projected MOE detector output data $\mathbf{z}_{1, l}, \quad l=1,2, \ldots, D$. These output data $z_{1, l}$ collected according to MMSE criteria are therefore used to extract the desired signal vector $\mathbf{x}(i)$ with $2 \times 1$ dimension. Let $\mathbf{V}$ be the $D \times 2$ weight matrix for the combining procedure, i.e.,

$$
\mathbf{x}(i)=\mathbf{V}^{H} \mathbf{z}(i)
$$

where the projected MIMO MOE detector output data vector $\mathbf{z}(i)$ is a $D \times 1$ vector 


$$
\mathbf{z}(i)=\left[\begin{array}{lll}
z_{1,1} & \cdots & z_{1, D}
\end{array}\right]^{T}=\mathbf{W}_{s}^{H} \widehat{\mathbf{y}}(i)
$$

The MMSE weight matrix can be determined by the following criteria

$$
\begin{aligned}
& \min _{\mathbf{V}_{1}} E\left\{\left|\mathbf{V}_{1}^{H} \mathbf{z}(i)-\mathbf{b}_{1}(i)\right|^{2}\right\} \\
& \min _{\mathbf{V}_{2}} E\left\{\left|\mathbf{V}_{2}^{H} \mathbf{z}(i)-\overline{\mathbf{b}}_{1}(i)\right|^{2}\right\}
\end{aligned}
$$

where $\mathbf{b}_{1}(i)$ and $\overline{\mathbf{b}}_{1}(i)$ in (9) are the training symbol sequences. This leads to the well-known Wiener solution

$$
\mathbf{V}_{1}=\mathbf{R}_{z z}^{-1} \tilde{\mathbf{H}}_{1}, \mathbf{V}_{2}=\mathbf{R}_{z z}^{-1} \tilde{\mathbf{H}}_{2}
$$

where $\mathbf{R}_{z z}=E\left\{\mathbf{z}(i) \mathbf{z}^{H}(i)\right\}$ is the $D \times D$ covariance matrix of the post-MOE data and $\tilde{\mathbf{H}}_{1}=E\left\{\mathbf{z}(i) \mathbf{b}_{1}^{H}(i)\right\}$ and $\tilde{\mathbf{H}}_{2}=$ $E\left\{\mathbf{z}(i) \overline{\mathbf{b}}_{1}^{H}(i)\right\}$ are the estimated composite channel matrices with $D \times 2$ dimensions.

\section{Complexity analysis}

This section analyzes and compares the computational complexity of the proposed fuzzy MOE method with conventional receivers such as $\mathrm{MOE}$ and subspace MOE detectors. For simplicity, only the number of complex multiplications is considered in the comparisons. Specifically, the proposed multistage detector scheme first calculates the computational complexity of coarse CFO estimator, fuzzy MOE detector, signal subspace projection, and MMSE weight combiner step-by-step. Summing the computational complexity of the multistage schemes then obtains the total computational complexity. Moreover, for the coarse CFO estimator, the number of complex multiplications needed to calculate the CFO shift spreading code matrix $\tilde{\mathbf{C}}_{1}(\Delta \varepsilon)$ in (14) is analyzed as follows

$$
N_{\mathbf{e}_{1}}=4 N^{2} L_{1}
$$

The number of complex multiplications of the spectral search algorithm in (15) is then computed as

$$
N_{S}=64 N K L_{1}+16 K^{2} L_{1}
$$

Second, for the fuzzy MOE detector, the number of complex multiplications of the weight matrix $\mathbf{W}$ of the MIMO fuzzy MOE detector in (18) is computed as

$$
N_{\mathrm{W}}=64 N^{3}+32 N^{2} D+8 N D^{2}+D^{3}
$$

where $\mathbf{R}_{\hat{y} \hat{y}}$ and $\mathbf{E}_{1}$ are the $4 N \times 4 N$ and $4 N \times D$ matrices, respectively. Next, the number of complex multiplications for signal subspace projection in (19) is computed as

$$
N_{\text {proj }}=32 N^{2} K+16 N^{2} D
$$

Finally, for MMSE weight combiner, the number of complex multiplications of the linear combiner in (20) and (21) is

$$
N_{\mathrm{LC}}=4 N D+2 D
$$

Then, the number of complex multiplications for the Wiener solution in (23) is

$$
N_{\text {Wiener }}=2\left(D^{3}+2 D^{2}\right)
$$

The total number of complex multiplications performed by the proposed multistage MOE detector in the above four stages can be summarized as

$$
\begin{aligned}
N_{\text {total }}= & N_{\tilde{C}_{1}}+N_{S}+N_{W}+N_{\text {proj }}+N_{\mathrm{LC}}+N_{\text {Wiener }} \\
= & 64 N^{3}+\left(4 L_{1}+32 K+48 D\right) N^{2} \\
& +\left(4 D+8 D^{2}+64 K L_{1}\right) N \\
& +\left(2 D+4 D^{2}+3 D^{3}+16 K^{2} L_{1}\right)
\end{aligned}
$$

According to (30), the computational complexity of the proposed multistage detector depends on the spreading code length $N$, the number of multipath channels $L_{1}$, the number of active users $K$, and the size $D$ of the dominant eigenvalues of the fuzzy constrained matrix $\mathbf{E}_{1}$. The equation also shows that the computational load is approximately $O\left(N^{3}\right)$. Note that the computational load of the proposed multistage detector is determined mainly by the weight matrix inversion calculated by the fuzzy MOE detector in (18).

Various existing methods are then used to evaluate the computational complexity of the conventional receivers, e.g., MOE and subspace MOE detectors are considered for comparison. The conventional MOE detector with $\mathbf{R}_{\hat{y} y}^{-1}$ as reported in [14] is considered first. The total number of multiplication operations is about $O\left(N^{3}\right)$ due to the major computations needed for inversion of $\mathbf{R}_{\widehat{y} \hat{y}}$. Next, the algorithm for the conventional subspace MOE detector in [17] resembles that for the projected weight matrix in (19). The major computations still require calculation of MOE weight by $\mathbf{R}_{y y}^{-1}$. Therefore, the number of complex multiplication is about $O\left(N^{3}\right)$. In summary, the complexity analysis shows that the total number of complex multiplication operations required by the proposed multistage detector, the conventional MOE, and subspace MOE detectors is about $O\left(N^{3}\right)$. That is, the MOE calculation results in a similar computational complexity in the three MOE calculation methods. 
Although the proposed multistage method requires additional computational operations, mainly because of the coarse CFO estimator and MMSE combiner, the computational load is still smaller than that of the MOE detector $O\left(N^{3}\right)$. As shown above, the proposed multistage detector has higher computational complexity compared to the conventional MOE and subspace MOE detectors. However, the simulation results confirm that proposed method outperforms the conventional MOE detector and confirms its robust performance over serious CFO environment.

\section{Computer simulations}

Simulations are performed to confirm the performance of the proposed MIMO MC-CDMA system over uplink multipath channels. For all users, the QPSK-modulated signals are spread by orthogonal gold codes of length 32 $(N=32)$. The channel profile assumes $L_{k}=4$ independent equal power Rayleigh fading paths with the time delays chosen from $\left\{0,3 T_{\mathrm{s}}\right\}$, which is smaller than the guard interval $T_{\mathrm{G}}=8 T_{\mathrm{s}}$ where $T_{\mathrm{s}}$ is the sampling period. In this simulation, the selected $D$ is $D=2 L_{1}$, i.e., two degrees of freedom are used to retain the desired user signal and to suppress MAI. The quasi-static multipath fading channel in the simulations is assumed to be constant during each packet and independent between packets. The fading gains are the i.i.d. complex Gaussian random variables with zero mean and unit variance. The output SINR, which is used as a performance index, is defined as the ratio of the signal power in $\mathbf{x}(i)$ to the MAI-puls-noise power in $\mathbf{x}(i)$ at the MMSE combiner output. The input SNR is also defined as SNR $=10 \log _{10}\left(\sigma_{1}^{2} / \sigma_{n}^{2}\right)$, and the near-far ratio (NFR) is defined as NFR $=10 \log _{10}\left(\sigma_{k}^{2} / \sigma_{1}^{2}\right)$, $k \neq 1$. The analysis is also simplified by assuming all MAI powers are identical. Executing 10,000 Monte-Carlo trials with different fading gains and data/noise sequences in each trial obtains one output SINR and BER value. The normalized CFOs of all users are also assumed to be in the range $-0.5<\Delta \varepsilon_{k}<0.5$. The performance comparison included the results obtained by conventional MOE, subspace MOE, and optimal MSINR detectors. The optimal MSINR receiver is implemented by using perfect CFO compensation and by artificially removing the desired user signal component in the data, and then using the ideal composite channel vectors $\tilde{\mathbf{h}}_{1}$ and $\tilde{\mathbf{h}}_{2}$ to obtain (7). Finally, the following "standard" parameters are used throughout the section unless otherwise mentioned: $\mathrm{SNR}=10 \mathrm{~dB}, \mathrm{NFR}=0 \mathrm{~dB}, K=10, \Delta \varepsilon_{1}=0.25$.

The first set of simulations evaluated the output SINR performance as a function of input SNR in the proposed multistage fuzzy MIMO MC-CDMA system. The simulation results in Figure 2 indicate that the performance of the proposed MIMO receiver with three processing stages and MMSE combiner approaches that of the ideal MSINR receiver. The proposed MOE detector outperforms conventional MOE and subspace MOE detectors, which are subject to the effects of CFO and MAI. Moreover, in a high-input SNR region, the first stage of the receiver, which has a coarse CFO estimator and MMSE combiner, also has a lower output SINR compared to the conventional MOE. Since the MAI power increases with input SNR in the NFR $=0 \mathrm{~dB}$ scenario, it performs poorly in CFO estimation. Thus, the MMSE combiner cannot effectively suppress MAI and induce output

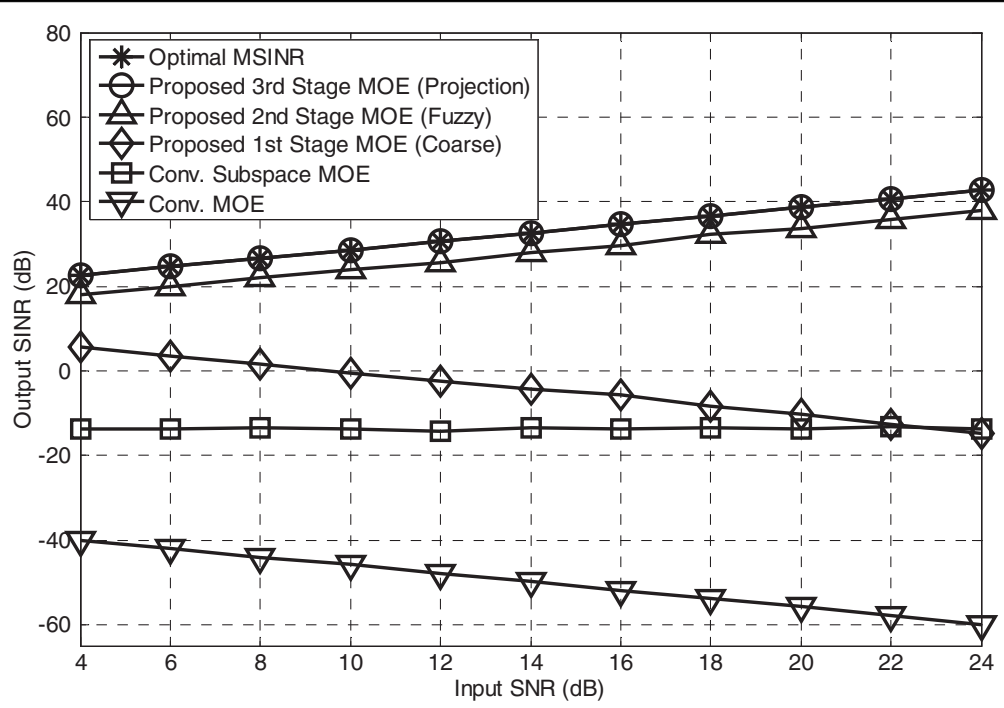

Figure 2 Output SINR comparison as a function of input SNR for MIMO MC-CDMA systems with $N=32, \mathrm{NFR}=0 \mathrm{~dB}, K=10$, and $\Delta \epsilon_{1}=0.25$. 


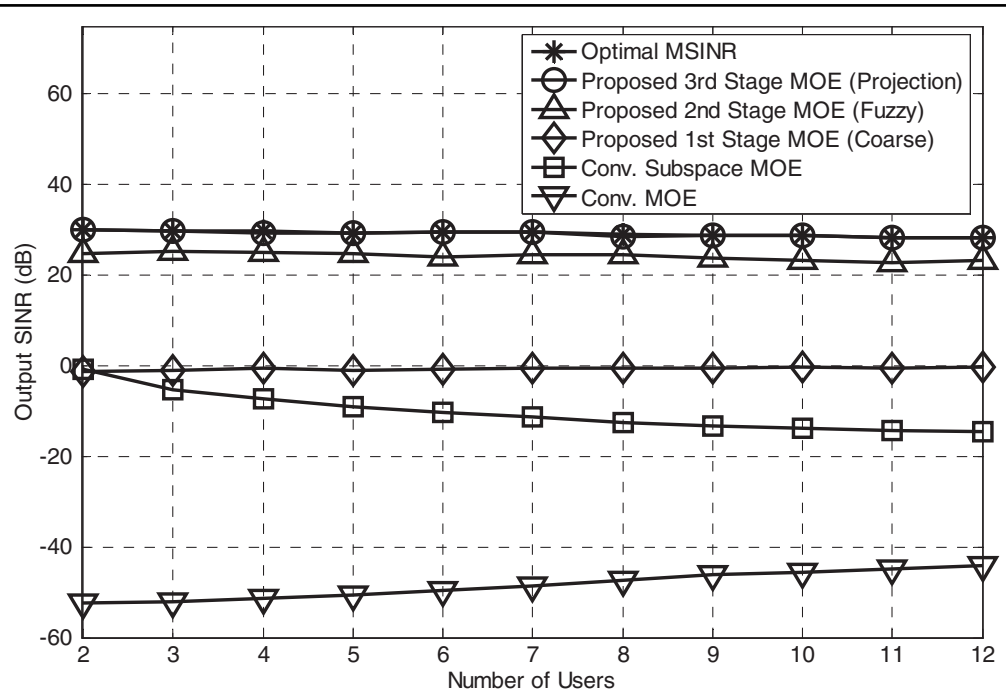

Figure 3 Output SINR comparison as a function of user number $K$ for MIMO MC-CDMA systems with $N=32, S N R=10 \mathrm{~dB}, \mathrm{NFR}=0 \mathrm{~dB}$, and $\Delta \epsilon_{1}=0.25$.

SINR degradation. The second stage with the coarse CFO estimator, fuzzy CFO-constrained MOE detector, and MMSE combiner performs robustly with only about $5 \mathrm{~dB}$ degradation due to the fuzzy CFO with large noise sequence. By using the full-stage processor and MMSE combiner described in Section 3, the performance of the third stage of the receiver approaches that of the ideal receiver as shown in Figure 2 since problematic noise is limited by the signal subspace projection. These results confirm that the proposed multistage MIMO MOE receiver indeed successfully suppresses MAI and effectively overcomes the CFO effect.
The second set of simulations compared the proposed MOE, conventional MOE, subspace MOE, and ideal MSINR receivers in terms of output SINR performance with increased numbers of users $K$. Figure 3 shows that, again, the MIMO multistage MOE detector successively approaches the ideal MIMO MSINR receivers and outperforms the conventional MOE and subspace MOE detectors. Moreover, the output SINRs of the conventional MOE receiver and first stage $\mathrm{MOE}$ receiver increase as the number of users increases. Because the power of MAI with different CFOs increases as the number of users increases, the MOE effectively

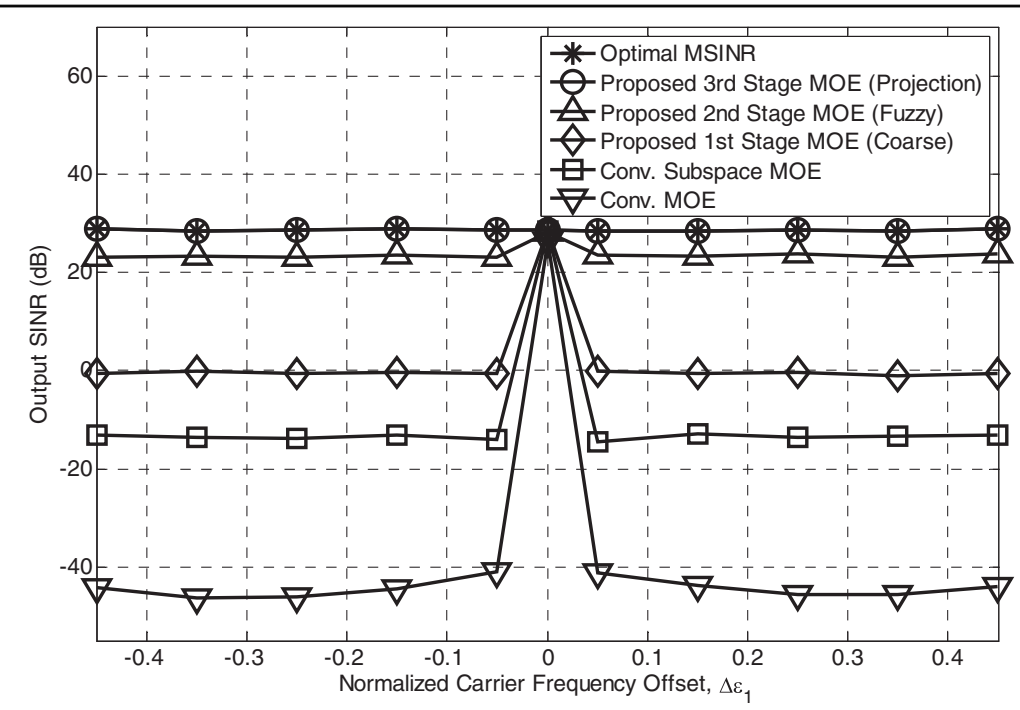

Figure 4 Output SINR comparison as a function of normalized CFO for MIMO MC-CDMA systems with $N=32, S N R=10 \mathrm{~dB}, \mathrm{NFR}=0 \mathrm{~dB}$, and $K=10$. 


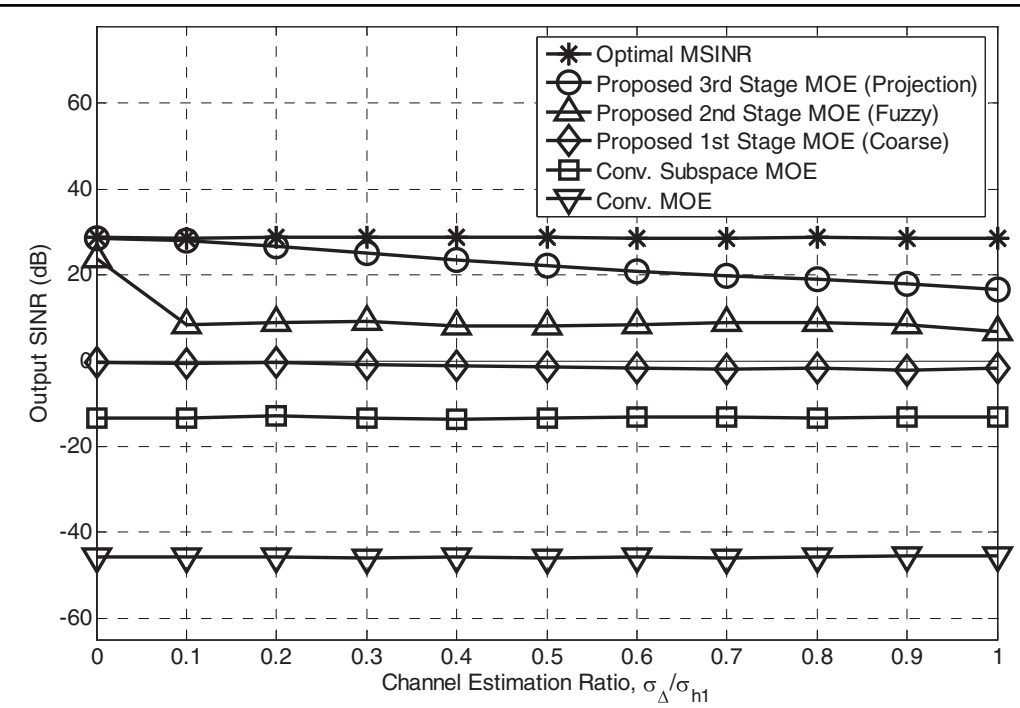

Figure 5 Output SINR comparison as a function of channel estimation ratio for MIMO MC-CDMA systems with $N=32, \mathrm{SNR}=10 \mathrm{~dB}$, $K=10$, and $\Delta \epsilon_{1}=0.25$.

suppress the MAI of overall CFO region. However, the output SINR performances of the conventional MOE and first stage MOE receivers are severely degraded in comparison with the proposed multistage and optimal receivers. Moreover, as the number of users increases, the proposed full-stage detector does not severely degrade the output SINR performance due to lack of degree of freedom effect, which is overcome by the larger code length.

The third set of simulations evaluated the tolerance of the proposed receiver to different frequency offsets. Figure 4 plots the resulting output SINR curves as a function of normalized CFO $\left(\Delta \varepsilon_{1}\right)$. Within the entire range
$-0.5<\Delta \varepsilon_{1}<0.5$, the output SINR of the proposed receiver is fairly constant. However, the conventional MOE and subspace MOE detectors exhibit severely degraded output SINR because of their extreme sensitivity to inaccuracies in frequency synchronization, which cause the desired signal cancellation. Therefore, Figure 4 indicates that the proposed multistage MOE detector with coarse CFO estimate, fuzzy CFO constraint, signal subspace projection, and MMSE combiner achieves the ideal performance and performs robustly against CFO by effectively collecting the desired signal and by canceling MAI interference. Notably, the performance at $\mathrm{CFO}=0$ case is better than other CFO cases. It is due to

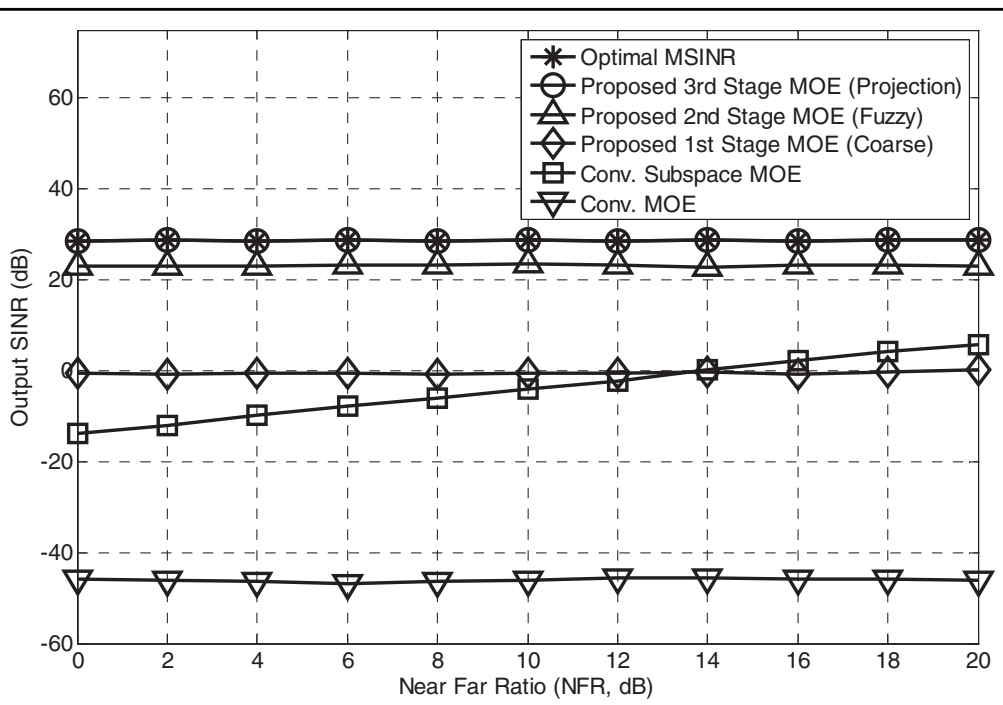

Figure 6 Output SINR comparison as a function of NFR for MIMO MC-CDMA systems with $N=32$, SNR=10 dB, $K=10$, and $\Delta \epsilon_{1}=0.25$. 


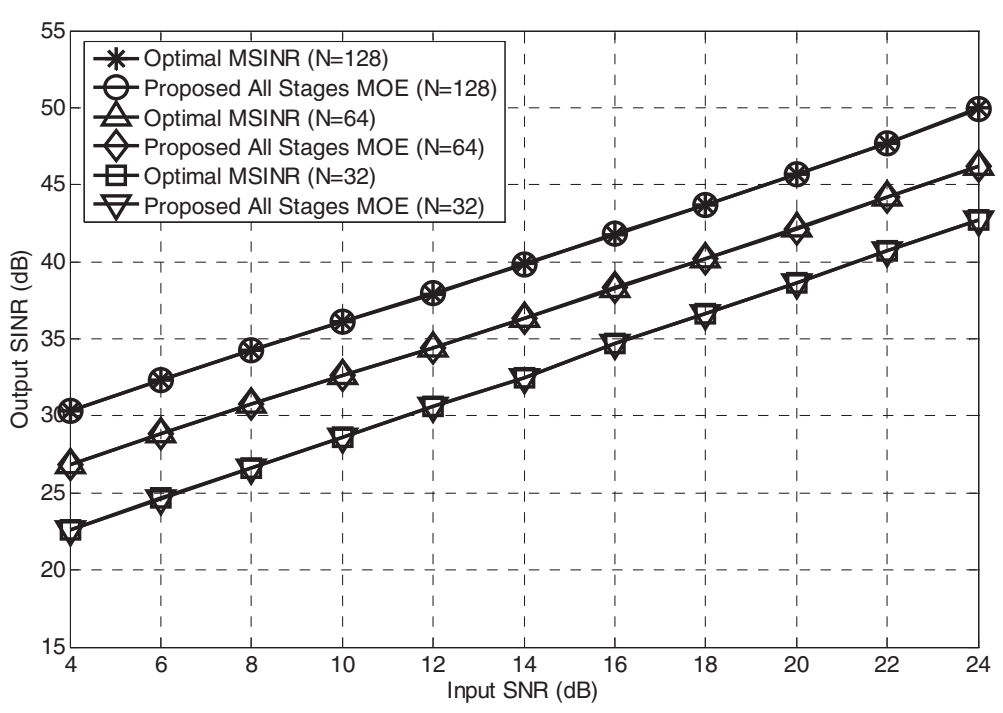

Figure 7 Output SINR performance comparison as a function of input SNR for MIMO MC-CDMA systems with different code length, $\mathrm{NFR}=0 \mathrm{~dB}, K=10$, and $\Delta \epsilon_{1}=0.25$.

no ICI interferences, which will be easily to suppress the MAI and get more degrees-of-freedom to enhance the output SINR performance.

The fourth set of simulations tested the robustness of the proposed MIMO MOE receiver against channel estimation error caused by time-varying fast fading channels. In this case, $\hat{\mathbf{h}}_{1}^{\left(n_{R}, n_{T}\right)}=\mathbf{h}_{1}^{\left(n_{R}, n_{T}\right)}+\sigma_{\Delta} \Delta \mathbf{h}$ is varied in (10) and (24), where $\Delta \mathbf{h}$ is a random vector with the entries being i.i.d. complex Gaussian random variables. Note that the entries of $\mathbf{h}_{1}$ are i.i.d. complex Gaussian random variables with variance $\sigma_{h 1}^{2}$. Figure 5 shows the output SINR versus $\sigma_{\Delta} / \sigma_{h 1}$. The simulation results confirm that the proposed MIMO MOE receiver performs reliably with relative error limited to $20 \%$ in channel estimation. Figure 5 also confirms that the proposed receiver outperforms other receivers in the channel estimation error scenario.

The fifth set of simulations investigated the near-far resistance of the proposed MIMO MOE detector under different NFR values. The output SINR results

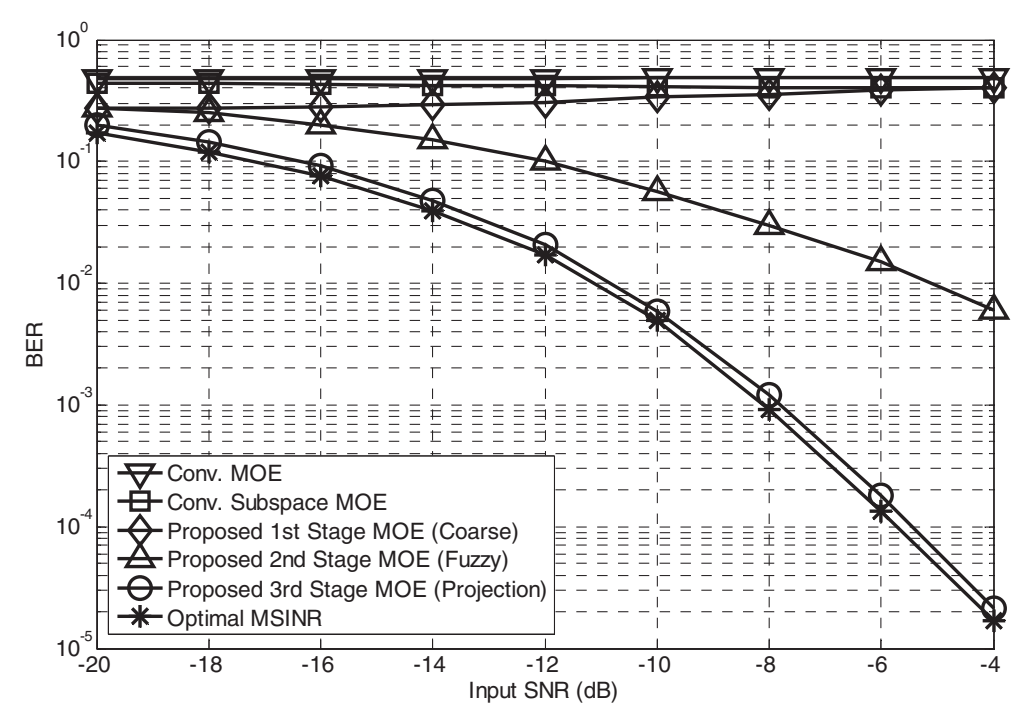

Figure 8 BER performance comparison as a function of input SNR for MIMO MC-CDMA systems with $N=32, N F R=0 \mathrm{~dB}, K=10$, and $\Delta \epsilon_{1}=0.25$. 
in Figure 6 show that the proposed robust MIMO MOE receivers provide excellent near-far resistance by multistage MAI cancellation. However, the subspace MOE detector outperforms the coarse method when the input SNR exceeds $14 \mathrm{~dB}$ because the strong MAI is suppressed by the signal subspace projection technique.

The sixth set of simulations investigated output SINR performance under varying code lengths. Figure 7 shows that, again, the proposed MIMO MOE receivers successively approach the ideal MIMO MSINR receiver performance for different code lengths. Moreover, the output SINR performance of the detector substantially improves with code length, which confirms that the proposed receiver is sufficiently robust to overcome CFO and MAI effects.

The final set of simulations investigated BER performance under varying input SNR. Figure 8 shows that the performance of the proposed MIMO MOE receiver again approaches that of the optimal MSINR receiver. In terms of BER, the proposed receiver performs much better compared to conventional MOE detectors, which confirms its superior robustness in suppressing CFO and MAI effects.

\section{Conclusions}

The novel robust MIMO MC-CDMA transceiver proposed in this study is suitable for uplink systems because of its high-quality performance over multipath fading channels and CFO effects. In the multiuser receiver, a MIMO fuzzy CFO-constrained MOE detector incorporating the rough CFO estimator suppresses MAI and overcomes the degradation problem of the conventional MOE detector with CFO effect. Next, by using signal subspace projection, the proposed detector further reduces noise with its fuzzy MOE filter. Finally, an MMSE weight combiner is proposed to capture the signal multipath diversity gain. Simulation results confirm that the proposed MIMO receiver performs comparably to the ideal receiver, outperforms conventional MOE detectors, and performs reliably over strong MAI and large frequency offsets.

\section{Competing interests}

The authors declare that they have no competing interests.

\footnotetext{
Acknowledgments

This study was sponsored by the National Science Council, R.O.C., under NSC contract 100-2220-E-155-006. The authors express the deepest gratitude to Prof. Tsui-Tsai Lin for his kind help and enthusiastic guidance. The authors also thank the Editor and anonymous reviewers for their helpful comments and suggestions in improving the quality of this article. Ted Knoy is appreciated for his editorial assistance.
}

Received: 3 November 2011 Accepted: 21 May 2012

Published: 21 June 2012

\section{References}

1. F.T. Chien, C.H. Hwang, C.C. Kuo, Performance analysis of multicarrier CDMA systems with frequency offsets and random spreading under optimum combining. IEEE Trans. Commun. 54(4), 737-747 (2006)

2. H. Cheng, S.C. Chan, Blind linear MMSE receivers for MC-CDMA systems. IEEE Trans Circ Syst I 54(2), 367-376 (2007)

3. R.W. Aldhaheri, A.H. Al-Qahtani, Performance analysis of fixed and mobile WiMAX MC-CDMA-based system, in IEEE ISWCS (, York, 2010), pp. 436-440

4. N. Langhammer, R. Reinhold, R. Kays, PHY modifications of IEEE 802.11 systems for transmission at very low SNR, in IEEE MC-SS (Herrsching, 2011), pp. 1-4

5. L. Tadjpour, S.H. Tasi, C.C. Kuo, Simplified multiaccess interference reduction for MC-CDMA with carrier frequency offsets. IEEE Trans. Veh. Technol. 59(5), 2543-2555 (2010)

6. S. Hara, R. Prasad, Design and performance of multicarrier CDMA system in frequency-selective Rayleigh fading channels. IEEE Trans. Veh. Technol. 48(5), 1584-1595 (1999)

7. D. Kivance, H. Liu, Uplink performance of MC-CDMA in the presence of frequency offset. in IEEE Veh. Technol. Conf. 5, 2855-2859 (1999). Amsterdam

8. J. Jang, K.B. Lee, Effects of frequency offset on MC/CDMA system performance. IEEE Commun. Lett. 3, 196-198 (1999)

9. Y. Zhao, S.G. Haggman, Sensitivity to Doppler shift and carrier frequency errors in OFDM systems-the consequences and solutions. in IEEE Veh. Technol. Conf. 3, 1564-1568 (1996). Atlanta

10. P.H. Moose, A technique for orthogonal frequency division multiplexing frequency offset correction. IEEE Trans. Commun. 42, 2908-2914 (1994)

11. J.D. Leclercq, Y. Zhao, S.G. Haggman, Intercarrier interference compression in OFDM communication systems by using correlative coding. IEEE Commun. Lett. 2, 214-216 (1998)

12. B. Seo, H.M. Kim, Frequency offset estimation and multiuser detection for MC-CDMA systems. in IEEE MILCOM 2002 2, 804-807 (2002). Anaheim, California

13. O. Besson, P. Stoica, On parameter estimation of MIMO flat-fading channels with frequency offsets. IEEE Trans. Signal Process. 51(3), 602-613 (2003)

14. H. Liu, Signal Processing Applications in CDMA Communications, Chapter 2 (Artech-House, London, 2000)

15. T. Pollet, M. Van Bladel, M. Moeneclaey, BER sensitivity of OFDM systems to carrier frequency offset and wiener phase noise. IEEE Trans. Commun. 43(2-4), 191-193 (1995)

16. X.D. Wang, H.V. Poor, Blind multiuser detection: a subspace approach. IEEE Trans. Inf. Theory 44(3), 677-690 (1998)

17. Z. Xu, P. Liu, X. Wang, Blind multiuser detection: from MOE to subspace methods. IEEE Trans. Signal Process. 52(2), 510-524 (2004)

18. S. Alamouti, A simple transmit diversity technique for wireless communications. IEEE J. Sel. Areas Commun. 16, 1451-1458 (1998)

19. Draft IEEE std 802.16e/D9, IEEE standard for local and metropolitan area networks-part 16: air interface for fixed and mobile broadband wireless access systems (, 2005)

20. S.P. Alex, L.M.A. Jalloul, Performance evaluation of MIMO in IEEE 802.16e/ WiMAX. IEEE Sel. Topics Signal Process 2(2), 181-190 (2008)

21. R. Van Nee, R. Prasad, OFDM Wireless Multimedia Communications (Artech House, London, 2000)

22. T.M. Lok, T.F. Wong, J.S. Lehnert, Blind adaptive signal reception for MCCDMA systems in Rayleigh-fading channels. IEEE Trans. Commun. 47, 464-471 (1999)

doi:10.1186/1687-1499-2012-197

Cite this article as: Deng and Liao: Fuzzy MIMO detector for MC-CDMA systems with carrier frequency offset over multipath fading channels. EURASIP Journal on Wireless Communications and Networking 2012 2012:197. 